\title{
Effectiveness of neonatal pulse oximetry screening for detection of critical congenital heart disease in daily clinical routine-results from a prospective multicenter study
}

\author{
Frank Thomas Riede • Cornelia Wörner • \\ Ingo Dähnert • Andreas Möckel • Martin Kostelka • \\ Peter Schneider
}

Received: 7 November 2009 / Accepted: 31 January 2010/Published online: 1 March 2010

(C) Springer-Verlag 2010

\begin{abstract}
Pulse oximetry screening (POS) has been proposed as an effective, noninvasive, inexpensive tool allowing earlier diagnosis of critical congenital heart disease (cCHD). Our aim was to test the hypothesis that POS can reduce the diagnostic gap in cCHD in daily clinical routine in the setting of tertiary, secondary and primary care centres. We conducted a prospective multicenter trial in Saxony, Germany. POS was performed in healthy term and post-term newborns at the age of 24-72 $\mathrm{h}$. If an oxygen saturation $\left(\mathrm{SpO}_{2}\right)$ of $\leq 95 \%$ was measured on lower extremities and confirmed after $1 \mathrm{~h}$, complete clinical examination and echocardiography were performed. POS was defined as false-negative when a diagnosis of cCHD was made after POS in the participating hospitals/at our centre. From July 2006-June 2008, 42,240 newborns from 34 institutions have been included. Seventy-two children were excluded due to prenatal diagnosis $(n=54)$ or clinical signs of cCHD $(n=18)$ before POS. Seven hundred ninetyfive newborns did not receive POS, mainly due to early discharge after birth $(n=727 ; 91 \%)$. In 41,445 newborns, POS was performed. POS was true positive in 14 , false positive in 40 , true negative in 41,384 and false negative in
\end{abstract}

F. T. Riede $(\bowtie) \cdot$ C. Wörner • I. Dähnert • M. Kostelka •

P. Schneider

Heart Center, University of Leipzig,

Strümpellstrasse 39,

04289 Leipzig, Germany

e-mail: frank-thomas.riede@med.uni-leipzig.de

\section{A. Möckel}

Department of Pediatrics, Helios Klinikum Borna,

Rudolf Virchow Strasse 2,

04552 Borna, Germany four children (three had been excluded for violation of study protocol). Sensitivity, specificity, positive and negative predictive value were $77.78 \%, 99.90 \%, 25.93 \%$ and $99.99 \%$, respectively. With POS as an adjunct to prenatal diagnosis, physical examination and clinical observation, the percentage of newborns with late diagnosis of cCHD was $4.4 \%$. POS can substantially reduce the postnatal diagnostic gap in cCHD, and false-positive results leading to unnecessary examinations of healthy newborns are rare. POS should be implemented in routine postnatal care.

Keywords Prenatal ultrasound - Physical examination . Pulse oximetry screening $\cdot$ Newborn .

Critical congenital heart disease

\section{Introduction}

Diagnosis and treatment of congenital heart disease have changed dramatically over the last few decades. The relative importance of preoperative morbidity and mortality has been increasing as a result of the great improvements in perioperative and long-term survival. This is especially true for critical congenital heart disease (cCHD), a group of morphologically heterogeneous disorders which have in common that early surgical or catheter interventional therapy is mandatory to achieve survival. Clinical examination is unable to detect all forms of cCHD [1, 32]. Heart murmurs, one of the hallmarks of non-critical heart disease typically diagnosed later in life may be absent or misleading because of the underlying anatomy, prolonged decline of pulmonary vascular resistance or reduced ventricular function. Despite the increasing use of 
prenatal diagnosis, a significant proportion of affected newborns is still not diagnosed before discharge after birth $[7,31]$. This problem may be aggravated by recent trends to earlier discharge and other changes in postnatal care [20]. The first manifestation of cCHD may be acute with circulatory collapse and the need for cardiopulmonary resuscitation or death, i.e. delayed diagnosis is associated with significant morbidity and mortality $[4,6,16,18]$. The current incidence of severe physiologic compromise resulting from previously unrecognised cCHD has been estimated to be 1 per 15,000 to 1 per 26,000 live births [27]. Hence, there is broad consensus that screening for cCHD is warranted [17, $27,29,30]$. However, it has been argued that "the use of prenatal ultrasound, close clinical observation during the transitional period and thorough physical examination alone" may be sufficient for timely diagnosis of cCHD [28]. This may be true in the specific setting of this single centre study, but the prerequisites mentioned are probably not encountered in the majority of hospitals or maternity units [25].

Pulse oximetry can detect mild hypoxemia, which is characteristic for many forms of cCHD, and may not be recognised by clinical examination [21]. The first abstracts examining pulse oximetry as a screening tool for cCHD were published in 1995 [5, 14]. Since then, there has been an increasing number of single and oligocentric studies on the subject $[2,3,13,15,19,23,24,26]$. Despite differences among the study protocols concerning target lesions, time of screening, cut-off values, probe placement and others, most authors came to the conclusion that pulse oximetry screening (POS) is a useful method. Recently published meta-analyses tend to share this view but argued that existing evidence is still insufficient to support a general recommendation for implementation of POS in routine care and therefore stated a need for further data [17, 29, 30].

The aim of our study was to test the hypothesis that POS, using currently available pulse oximeters, can effectively reduce the diagnostic gap in cCHD in daily clinical routine in centres of primary, secondary and tertiary care.

\section{Materials and methods}

A prospective multicenter field study of 34 neonatal/obstetrical departments in Saxony, Germany, was performed from July 2006 to June 2008. All full and post-term neonates (gestational age $\geq 37$ weeks) meeting the following criteria were included in the study: (1) no prenatal diagnosis/suspicion of congenital heart disease, (2) normal routine clinical examination (3) informed parental consent.

All forms of cCHD were defined as the target of POS, regardless of whether cyanosis, i.e. duct dependency, is an invariable or a possible feature of the particular condition (Table 1).
Table 1 Types of critical congenital heart disease (cCHD) defined as target lesions for pulse oximetry screening (POS)

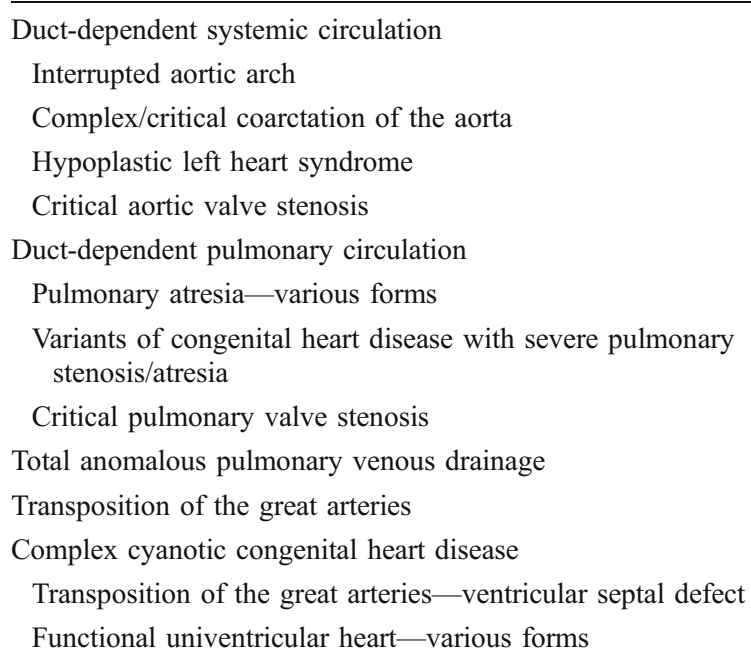

At the age of 24-72 h, POS was performed by midwives, nurses and physicians experienced in the routine use of pulse oximetry. No particular requirements concerning the type of pulse oximeter were made so that a great variety of devices was used. All pulse oximeters measured functional oxygen saturation $\left(\mathrm{SpO}_{2}\right)$. The probe was placed on either foot. A $\mathrm{SpO}_{2}$ of $\geq 96 \%$ was defined as normal. POS was positive when a $\mathrm{SpO}_{2}$ of $\leq 95 \%$ was measured and confirmed after $1 \mathrm{~h}$. POS results were recorded in each newborn's hospital chart as well as in its individual booklet for prevention during childhood and adolescence ("Gelbes Heft"). A positive POS was followed by complete clinical examination and echocardiography. After negative POS, no further actions were taken. POS was defined as false-negative when a diagnosis of cCHD was made after POS in the participating hospitals/at our centre.

Data acquisition was performed at the study sites, at the central office for quality management in perinatal and neonatal care of the Saxonian Medical Association and at our hospital, which is the referral centre for participants of the study. Data analysis and descriptive statistics were performed using commercially available software (Microsoft ${ }^{\circledR}$ Excel $^{\circledR} 2008$ for Mac). Sensitivity, Specificity, positive and negative predictive values for POS were calculated.

The study protocol was approved by the Ethics Committee of the University of Leipzig, Germany.

\section{Results}

During the study period, there were 48,348 live births in the participating hospitals. A total of 6,108 newborns were excluded from the study. Of these, 54 had prenatal diagnosis of cCHD. In 18 of them, clinical symptoms led 
to the diagnosis of cCHD before POS. A total of 6,036 newborns received diagnosis/treatment for other conditions. No parents refused to participate in the study.

Newborns eligible for POS, 42,240 of them, were included in the study. Seven hundred ninety-five did not receive POS, mainly because of early discharge after birth $(n=727 ; 91 \%)$. A total of 41,445 newborns received POS. Three children were excluded from further analysis because the study protocol had not been correctly followed. Pulse oximetry had been performed in these three early on the first day of life (i.e. at an age of 4, 6 and $10 \mathrm{~h}$, respectively) resulting in $\mathrm{SpO}_{2}$ ranging from $94 \%$ to $95 \%$ at initial and control measurement.
All were discharged early (one after exclusion of cCHD by echocardiography), and none was re-admitted for cCHD. A $\mathrm{SpO}_{2}$ of $\leq 95 \%$ was measured in $65(0.16 \%)$ of 41,442 neonates. This measurement was confirmed, i.e. POS was positive in $54(0.13 \%)$ of 41,445 neonates. POS was falsepositive related to the diagnosis of cCHD in 40 newborns (healthy $n=12$, persistent pulmonary hypertension of the newborn $n=15$, sepsis $n=13$ ). POS was true-positive in 14 children (Fig. 1 and Table 2). The types of cCHD detected by POS are listed in Table 2. POS was true-negative in 41,384 and false-negative in four children (Table 3 ). The latter were diagnosed at the age of 3-8 days before $(n=1)$ and after

Fig. 1 Flow chart of the study population

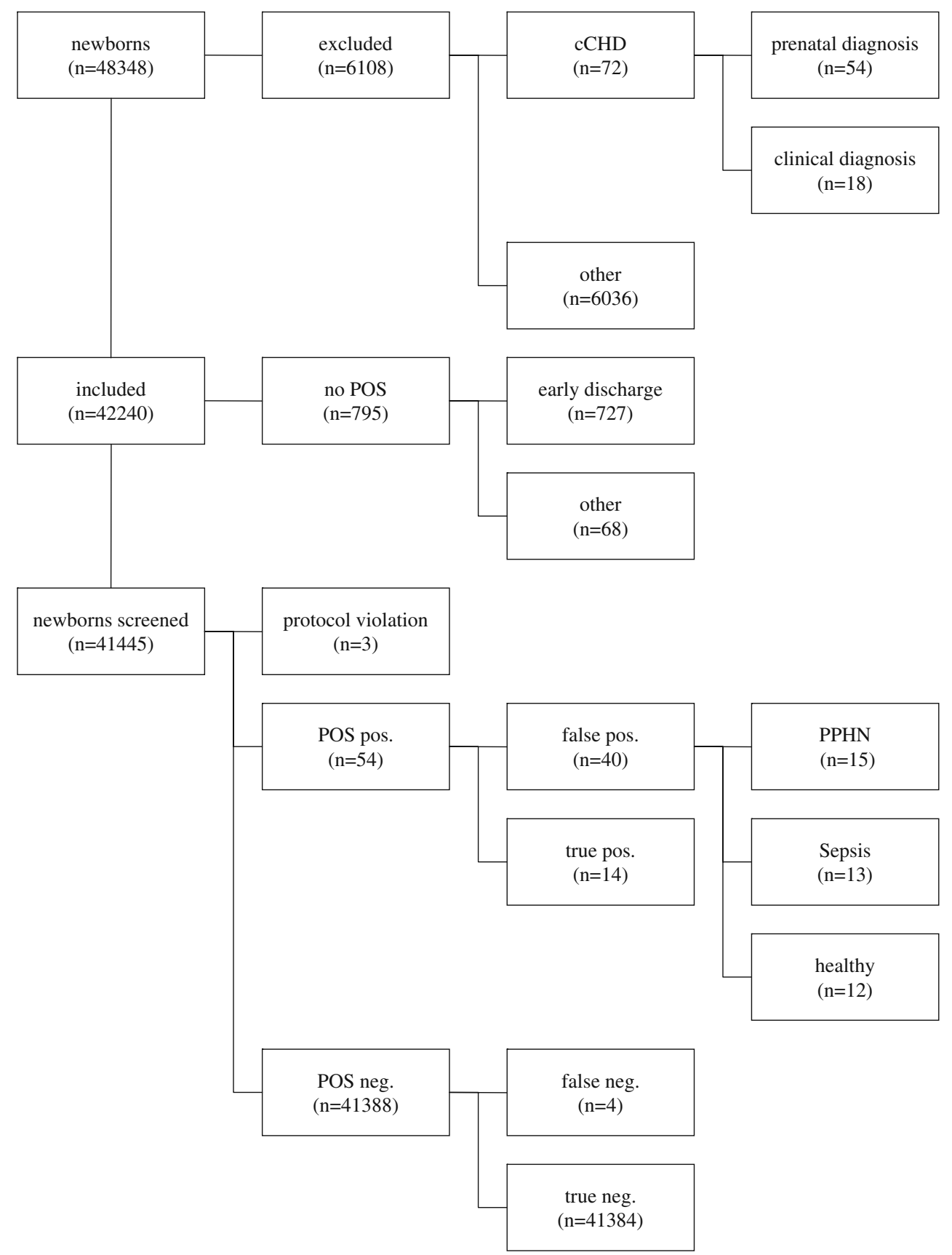


Table 2 Types of critical congenital heart disease (cCHD) detected by pulse oximetry screening (POS) $(n=14)$

\begin{tabular}{ll} 
Duct-dependent systemic circulation & 2 \\
Persistent truncus arteriosus, interrupted aortic arch & 1 \\
Hypoplastic left heart syndrome & 1 \\
Duct-dependent pulmonary circulation & 2 \\
Pulmonary atresia, ventricular septal defect & 5 \\
Total anomalous pulmonary venous drainage & 2 \\
Transposition of the great arteries & 3 \\
Complex cyanotic congenital heart disease & 2 \\
Transposition of the great arteries-ventricular septal defect & \\
Taussig-Bing syndrome & 1 \\
\hline
\end{tabular}

$(n=3)$ discharge from the maternity unit. The patient with transposition of the great arteries and ventricular septal defect presented with dyspnea and cyanosis: prostaglandin was infused. The patients with coarctation had severe circulatory impairment necessitating catecholamine support and/or mechanical ventilation. All were transferred to our hospital and subsequently underwent successful cardiac surgery.

Sensitivity, specificity, positive and negative predictive value for POS were $77.78 \%, 99.90 \%, 25.93 \%$ and $99.99 \%$, respectively.

The incidence of cCHD $(n=90)$ in the study population $(n=48,348)$ was $0.186 \%$. The modes of diagnosis are summarised in Fig. 2. In the perinatal and neonatal registries, maintained at the Saxonian Medical Association, there was no death resulting from cCHD outside of our centre, i.e. any being related to delayed diagnosis or nonreferral among our study population.

\section{Discussion}

The total number of patients, in whom POS has been assessed for earlier detection of cCHD exceeds 150,000 [9, 17]. Our study differs from most of the previously published reports mainly in that we included 42,240 newborns, which is comparable only to recent data from Meberg and de-Wahl Granelli [9, 19]. Furthermore, a broad spectrum of hospitals providing primary, secondary or tertiary care participated in our study with the aim of examining whether POS is able to reduce the diagnostic

Table 3 Types of critical congenital heart disease (cCHD) missed by pulse oximetry screening (POS) $(n=4)$

\begin{tabular}{|c|c|}
\hline Duct-dependent systemic circulation & 3 \\
\hline Critical coarctation of the aorta & 2 \\
\hline Complex coarctation of the aorta-ventricular septal defect & 1 \\
\hline Complex cyanotic congenital heart disease & 1 \\
\hline Transposition of the great arteries - ventricular septal defect & 1 \\
\hline
\end{tabular}

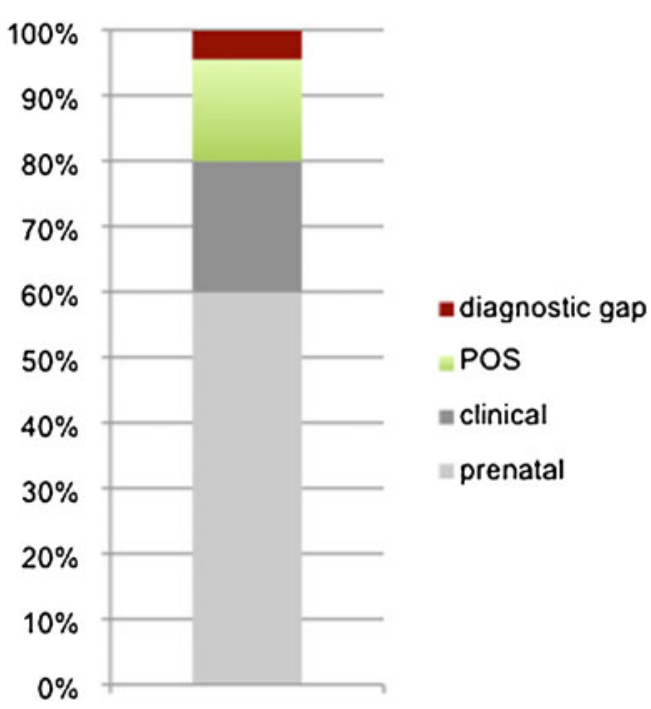

Fig. 2 Reduction of the "diagnostic gap" in critical congenital heart disease (cCHD) with pulse oximetry screening. All newborns with critical cCHD $(n=90$ out of the study population $(n=48,348))$ are shown. Colours indicate in percentages whether the diagnosis was made by prenatal ultrasound (prenatal), by physical examination or clinical observation (clinical) before pulse oximetry screening, i.e. during the first $24 \mathrm{~h}$ of life or by pulse oximetry screening (POS). When POS was used, only $4.4 \%$ of newborns had a delayed diagnosis of cCHD (diagnostic gap)

gap in cCHD not only in controlled single or oligocentric studies but also under "real life" conditions.

In our study, there was a relatively high percentage of cCHD detected by prenatal ultrasound $(60 \%)$. It would be of interest to know which results would have been obtained by POS in these newborns. However, when a prenatal diagnosis of cCHD is made, perinatal management is planned as soon as parents opt for postnatal therapy (and not for abortion or compassionate care). After delivery, these newborns receive immediate medical assessment and treatment if the diagnosis is confirmed. Treatment frequently consists of prostaglandin infusion and volume replacement and may include catheter interventions (balloon atrioseptostomy of a restrictive foramen ovale, balloon angioplasty of critical valvular stenoses and, rarely, stenting of restrictive communications at the atrial or ductal level). $\mathrm{SpO}_{2}$ is an important target in the postnatal management of cCHD. It is kept within the normal range in non-cyanotic lesions, whereas in many cyanotic lesions, an attempt is made to maintain pulmonary to systemic flow ratio at $1: 1$ (corresponding to an $\mathrm{SpO}_{2}$ of about $80 \%$ ). Thus, $\mathrm{SpO}_{2}$ in these newborns rather reflects the underlying hemodynamics and the quality of postnatal care. It does not answer the question whether these children would have been detected by POS (or by previous clinical observation/physical examination). This would only have been possible if the prenatal diagnosis and postnatal care had been withheld, which obviously was not feasible for ethical reasons. 
After the first physical examination and clinical observation during the first $24 \mathrm{~h}$ of life, i.e. before POS was performed, $80 \%$ of all cCHD had been diagnosed. A second physical examination before discharge from maternity is current practice in our as well in many other regions. However, this does not seem to increase the sensitivity which can be achieved with this method [10]. Thus, it seems reasonable to assume that the diagnostic gap without POS would have remained at $20 \%$, which is in agreement with the data reported by Wren [31]. Using POS to complement prenatal ultrasound and physical examination/ clinical observation achieved a diagnostic gap as low as $4.4 \%$ (Fig. 2). We believe that this number is more important than the sensitivity of POS alone, which was $77.78 \%$ in our study and is comparable to other large series $[9,15,19]$. This relates to the fact that the reduction of the diagnostic gap in cCHD reflects the aim of POS - at least from a clinician's perspective. Unlike other methods, for example, newborn metabolic or hearing screening POS is not meant as a new test for more or less replacing other existing methods. Rather, it can be thought of as a tool which is complementary to prenatal ultrasound, physical examination and clinical observation. It has been well documented that POS sometimes does not detect left heart obstructive lesions which represented $75 \%$ of the falsenegative cases in our series [30]. This is not surprising, as for example coarctation of the aorta may develop and progress early after birth and is not always duct-dependent. Nevertheless, in some cases, there is right to left shunting via a patent arterial duct; for this reason we did not exclude these lesions from the target group. We would like to underscore the idea that POS is a complementary tool and that thorough physical examination should not only include the search for signs of heart failure but also comparative pulse palpation. Complex cyanotic lesions can be also be missed by POS alone, especially in the context of high pulmonary blood flow. However, concomitant clinical examination may detect tachypnea and prompt further evaluation [12].

Not surprisingly, sensitivity and reliability of POS may also be influenced by human factors, and the training level of the staff performing POS should be adequate [22].

The rate of false-positive results in our study was very low $(0.10 \%)$. Others reported similar results $[9,15]$. Furthermore, some of these neonates had other diseases, like neonatal sepsis, for which earlier diagnosis does no harm. It has also been shown that earlier screening leads to a higher percentage of false-positive results [19]. Therefore, most publications, including one large review, recommend that POS be performed at or after $24 \mathrm{~h}$ of age $[9,15,30]$.

It has been argued that POS should be done with a newgeneration pulse oximeter [8]. On the other hand, existing technology is used to monitor critically ill children and to guide intensive care and theoretical differences may not always be clinically relevant [30]. In our study - undertaken with a great variety of pulse oximeters - the percentage of cCHD diagnosed before discharge from maternity was even higher than in the Swedish study, where newgeneration pulse oximeters were used (95.6 vs. 92\%) [9].

Most authors currently use a high cut-off value of $95 \%$ or $96 \%$, at which level POS seems to have the best overall performance $[2,9,15,19,23,24,26]$. Between these two values, differences are probably minimal, and with a higher cut-off, the need for measurement of pre- and postductal difference in $\mathrm{SpO}_{2}$ decreases [30]. However, postductal measurement is important, as some forms of cCHD associated with predominant right to left shunting at ductal level may be missed when only preductal $\mathrm{SpO}_{2}$ is used.

Our study protocol included repeated $\mathrm{SpO}_{2}$ measurements after $1 \mathrm{~h}$ if the initial value was $<96 \%$. This reduced the number of newborns undergoing clinical examination and echocardiography from 65 to 54 . After having accumulated more data, further analyses may show that in newborns with an $\mathrm{SpO}_{2}$ below a certain threshold, assessment should be initiated directly without repeated measurements.

In our study, we did not address the issue of costs which has been extensively examined by Griebsch et al. who came to the conclusion that POS is at least cost-neutral in the short term [11]. Taking into account the financial burden of death and sequelae of neurologic impairment or disability, which may result from late diagnosis of cCHD, POS should be cost effective [29, 30].

In reviewing our data and the experience published by others, we agree that there remain some open questions concerning the use of POS. On the other hand, using currently available pulse oximeters POS as an adjunct to prenatal ultrasound, thorough physical examination and clinical observation can substantially reduce the diagnostic gap in cCHD. In view of the persisting problem of late diagnosis of cCHD and its potential sequelae, we think it is no longer justified to withhold this method. We would rather suggest that POS be implemented in routine neonatal care while scientific evaluation of the method is continued to allow for modifications. In Saxony, after the end of our study, an appropriate recommendation has been put forward by the Working Groups for Perinatology and Neonatology of the Saxonian Medical Association.

\section{Limitations of the study}

During the study period, there were no reports of suspected death from unrecognised cCHD from the participating hospitals. In the Saxonian perinatal and neonatal registries, there were no deaths in external hospitals resulting from cCHD among our study population. Due to statutory provisions, there is no compulsory autopsy in our region. 
We, therefore, cannot fully exclude single missed cases of death from undiagnosed cCHD. Additionally, after discharge from a maternity unit, there may have been readmissions for cCHD in hospitals in other regions, i.e. not covered by Saxonian registries. However, the incidence of cCHD in our population was rather high compared to what should be expected from published reports. We can therefore assume that any potential resulting error is small.

Acknowledgements We would like to thank all of the participants in our study for their continuous cooperation and support, which made this work possible. A detailed list of all the institutions is given below.

Klinik für Kinder-und Jugendmedizin, HELIOS Klinikum Aue $\mathrm{GmbH}$, ChA PD Dr. med. habil. G. Frey, Ansprechpartner: Fr. OÄ Dr. med. S. Kurzweg, Gartenstraße 6, 08280 Aue

Klinik f. Kinder-u. Jugendmedizin, Krankenhaus Bautzen, OberlausitzKliniken gGmbH, ChA Dr. med. U. Winkler, Ansprechpartner: Hr. Dr. med. T. Zimmermann, Am Stadtwall 3, 02625 Bautzen

Klinik für Kinder-und Jugendmedizin, HELIOS Klinik Borna (Leipziger Land), ChA Dr. med. A. Möckel, Rudolf-Virchow-Straße 2, 04552 Borna

Klinik für Kinder-und Jugendmedizin, DRK Krankenhaus Chemnitz-Rabenstein, ChÄ Dr. med. I. Ruschke, Unritzstraße 23, 09117 Chemnitz

Frauenklinik, Krankenhaus Dresden-Friedrichstadt, Städtisches Klinikum, ChA Dr. med. K.W. Degen, Ansprechpartner Neonatologie: Leitender Pädiater Hr. DM W. Möhr, Friedrichstraße 41, 01067 Dresden

Zentrum für Kinder-und Jugendmedizin, Städtisches Krankenhaus Dresden-Neustadt, ChÄ Prof. Dr. med. habil. M. Kabus, Ansprechpartner: Hr. OA Dr. med. N. Lorenz, Industriestraße 40, 01129 Dresden

Klinik für Gynäkologie und Geburtshilfe, Krankenhaus St. JosephStift Dresden, ChA Dr. med. K. Wagner, Ansprechpartner: Fr. Dr. med. B. Friedrich, Wintergartenstraße 15-17, 01307 Dresden

Klinik für Frauenheilkunde und Geburtshilfe, Universitätsklinikum Carl Gustav Carus, TU Dresden, Direktor Prof. Dr. med. habil. W. Distler, Ansprechpartner: Fr. OÄ Dr. med. G. Kamin, Fetscherstra $\beta$ e 74, 01307 Dresden

Klinik für Gynäkologie und Geburtshilfe, Klinik Eilenburg, Kreiskrankenhaus Delitzsch GmbH, ChA Doz. Dr. med. H. Gstöttner, Wilhelm-Grune-Straße 5-8, 04838 Eilenburg

Klinik für Kinder-und Jugendmedizin, Kreiskrankenhaus Freiberg gGmbH, ChA Dr. K.D. Paul, Ansprechpartner: Fr. Dr. med. K. Schmidt, Station K 1-Neonatologie, Donatsring 20, 09599 Freiberg

Klinik für Kinder-und Jugendmedizin, Krankenhaus Freital, Weißeritztal-Kliniken GmbH, ChA Dr. med. G. Heubner, Bürgerstraße 7, 01705 Freital

Klinik für Gynäkologie und Geburtshilfe, Krankenhaus Grimma, Muldentalkliniken GmbH, ChA Dr.med. U. Piskazeck, Kleiststraße 5, 04668 Grimma

Klinik für Frauenheilkunde und Geburtshilfe, Elblandklinikum Großenhain, Elblandkliniken Meißen GmbH \& Co. KG, ChA Dr. med. J. Kotsch, Weinbrunnenstraße 15, 01558 Großenhain

Klinik für Gynäkologie und Geburtshilfe, Diakoniekrankenhaus Chemnitzer Land, DIAKOMED gGmbH, ChÄ DM U. Hensel, Ansprechpartner: Hr. Dr. med. E. Frömmel, Limbacher Straße 19b, 09232 Hartmannsdorf

Klinik für Kinder-und Jugendmedizin, Klinikum Hoyerswerda, ChÄ Dr. med. P. Jesche, Maria-Grollmu $\beta$-Straße 10, 02977 Hoyerswerda Geburtshilfe und Pränatalmedizin, St. Elisabeth-Krankenhaus Leipzig, ChA Dr. med. C. Springer, Biedermannstraße 84, 04277 Leipzig

Klinik für Kinder-und Jugendmedizin, Städtisches Klinikum "St. Georg" Leipzig, ChA Prof. Dr. med. habil. T. Richter, Ansprechpartner: Fr. OÄ Dr. med. B. Linse, Neonatologie, Delitzscher Straße 141, 04129 Leipzig
Universitätsklinik und Poliklinik für Kinder und Jugendliche, Universitätsklinikum Leipzig AöR, Direktor: Prof. Dr. W. Kiess, Ansprechpartner: Fr. Dr. med. N. Wolf, Neonatologische Intensivstation, Liebigstraße 20a, 04103 Leipzig

Klinik für Kinder-und Jugendmedizin, HELIOS Krankenhaus Leisnig, ChA PD Dr. med. habil. P. Müller, Colditzer Straße 48, 04703 Leisnig

Klinik für Kinderheilkunde, Elblandklinikum Meißen, Elblandkliniken Meißen GmbH \& Co. KG, ChA Dr. med. K.N. Pargac, Nassauweg 7, 01662 Meißen

Abteilung für Frauen-und Geburtsheilkunde, Krankenhaus Mittweida, Landkreis Mittweida Krankenhaus gGmbH, ChA Dr. med. G. Fischer, Ansprechpartner: Fr. OÄ DM S. Braun, Geburtshilfe Station 5, Hainichener Straße 4-6, 09648 Mittweida

Klinik f. Kinder-und Jugendmedizin, Außenstelle des Universitätsklinikums Dresden, Klinikum Pirna GmbH, ChA Dr. med. G. Heubner, Struppener Straße 13, 01796 Pirna

Klinik für Kinder-und Jugendmedizin, HELIOS VogtlandKlinikum Plauen GmbH, ChA Dr. med. J. Quietzsch, Ansprechpartner: Fr. OÄ Dr. med. S. Andiel, Röntgenstraße 2, 08529 Plauen

Klinik für Frauenheilkunde, Elblandklinikum Radebeul, Elblandkliniken Meißen GmbH \& Co. KG, ChÄ Dr. med. B. Richter, Heinrich-Zille-Straße 13, 01445 Radebeul

Abteilung Gynäkologie/Geburtshilfe, Paracelsus-Klinik Reichenbach, Leitende Ärztin Fr. DM U. Köhler, Plauensche Straße 37, 08468 Reichenbach

Klinik f. Kinder-und Jugendmedizin, Klinikum Obergöltzsch Rodewisch, ChA DM R. Hanusch, Stiftstraße 10, 08228 Rodewisch Abteilung Geburtshilfe/Gynäkologie, HELIOS Klinik Schkeuditz, ChA Dr. med. B. Stubert, Anpsrechpartner: Hr. DM W. Richter, Leipziger Straße 45, 04435 Schkeuditz

Klinik für Gynäkologie und Geburtshilfe, Kreiskrankenhaus Stollberg gGmbH, ChA DM M. El Kaissi, Jahnsdorfer Straße 7, 09366 Stollberg

Klinik für Kinder-und Jugendmedizin, Kreiskrankenhaus Torgau, "Johann Kenntmann" gGmbH, ChA Dr. med. W. Pernice, Ansprechpartner: Fr. OÄ DM Edith Bieck, Neugeborenen und Kinderabteilung, Christianistraße 1, 04860 Torgau

Abteilung für Kinder-und Jugendmedizin, Kreiskrankenhaus Wei $\beta$ wasser gGmbH, ChÄ Dr. med. B. Niemz, Karl-Liebknecht-Straße 1, 02943 Wei $\beta$ wasser

Abteilung Kinder-und Jugendmedizin, Pleißental-Klinik GmbH, ChA Dr. med. D. Ermisch, Ansprechpartner Hr. OA Dr. med. A. Geißen, Ronneburger Straße 106, 08412 Werdau

Abteilung für Kinder-und Jugendmedizin, Krankenhaus Wurzen, Muldentalkliniken GmbH, ChÄ Dr.med. K. Gröger, Kutusowstraße 70, 04808 Wurzen

Klinik für Kinder-und Jugendmedizin, Klinikum des Landkreises Löbau-Zittau gGmbH, ChA Dr. med. T. Verbeek, Ansprechpartner: Fr. OÄ MUDr. H. Reck, Görlitzer Straße 8, 02763 Zittau

Klinik für Kinder-und Jugendmedizin, Heinrich-Braun-Klinikum Zwickau gGmbH, ChA Dr. med. T. Stuckert, Ansprechpartner: Fr. OÄ Dr. med. Troeger-Reinfried, Karl-Keil-Straße 35, 08060 Zwickau

We are also grateful to Ms. Annette Kaiser and Ms. Annette Friedrich (of the central office for quality management in perinatal and neonatal care of the Saxonian Medical Association) for providing data from the Saxonian perinatal and neonatal registries.

Conflict of interest The authors declare that they have no conflict of interest.

\section{References}

1. Ainsworth S, Wyllie JP, Wren C (1999) Prevalence and clinical significance of cardiac murmurs in neonates. Arch Dis Child Fetal Neonatal Ed 80:F43-F45 
2. Arlettaz R, Bauschatz AS, Mönkhoff M, Essers B, Bauersfeld U (2006) The contribution of pulse oximetry to the early detection of congenital heart disease in newborns. Eur J Pediatr 165:94-98

3. Bakr AF, Habib HS (2005) Combining pulse oximetry and clinical examination in screening for congenital heart disease. Pediatr Cardiol 26:832-835

4. Brown KL, Ridout DA, Hoskote A, Verhulst L, Ricci M, Bull C (2006) Delayed diagnosis of congenital heart disease worsens preoperative condition and outcome of surgery in neonates. Heart 92:1298-1302

5. Byrne BJ, Donohue PK, Bawa RD et al (1995) Oxygen saturation as a screening test for critical congenital heart disease [abstract]. Pediatr Res 37(Suppl):198A

6. Chang RK, Gurvitz M, Rodriguez S (2008) Missed diagnosis of critical congenital heart disease. Arch Pediatr Adolesc Med 162:969-974

7. Chang RK, Rodriguez S, Klitzner TS (2009) Screening newborns for congenital heart disease with pulse oximetry: survey of pediatric cardiologists. Pediatr Cardiol 30:20-25

8. de Wahl Granelli A, Mellander M, Sunnegårdh J, Sandberg K, Ostman-Smith I (2005) Screening for duct-dependant congenital heart disease with pulse oximetry: a critical evaluation of strategies to maximize sensitivity. Acta Paediatr 94:1590-1596

9. e Wahl Granelli A, Wennergren M, Sandberg K, Mellander M, Bejlum C, Inganäs L, Eriksson M, Segerdahl N, Agren A, EkmanJoelsson BM, Sunnegårdh J, Verdicchio M, Ostman-Smith I (2009) Impact of pulse oximetry screening on the detection of duct dependent congenital heart disease: a Swedish prospective screening study in 39, 821 newborns. BMJ 338:a3037

10. Glazener CM, Ramsay CR, Campbell MK, Booth P, Duffty P, Lloyd DJ, McDonald A, Reid JA (1999) Neonatal examination and screening trial (NEST): a randomised, controlled, switchback trial of alternative policies for low risk infants. BMJ 318:627-631

11. Griebsch I, Knowles RL, Brown J, Bull C, Wren C, Dezateux CA (2007) Comparing the clinical and economic effects of clinical examination, pulse oximetry, and echocardiography in newborn screening for congenital heart defects: a probabilistic costeffectiveness model and value of information analysis. Int $\mathrm{J}$ Technol Assess Health Care 23(2):192-204

12. Hetzel PG, Glanzmann R, Günthard J, Bruder E, Godi E, Bührer C (2007) Failed detection of complex congenital heart disease (including double outlet right ventricle and total anomalous pulmonary venous return) by neonatal pulse oximetry screening. Eur J Pediatr 166:625-626

13. Hoke TR, Donohue PK, Bawa PK, Mitchell RD, Pathak A, Rowe PC, Byrne BJ (2002) Oxygen saturation as a screening test for critical congenital heart disease: a preliminary study. Pediatr Cardiol 23:403-409

14. Kao BA, Felt LR, Werner JC (1995) Pulse oximetry as a screen for congenital heart disease in newborns [abstract]. Pediatr Res 37:216A

15. Koppel RI, Druschel CM, Carter T, Goldberg BE, Mehta PN, Talwar R, Bierman FZ (2003) Effectiveness of pulse oximetry screening for congenital heart disease in asymptomatic newborns. Pediatrics 111:451-455

16. Kuehl KS, Loffredo CA, Ferenez C (1999) Failure to diagnose congenital heart disease in infancy. Pediatrics 103:743-747
17. Mahle WT, Newburger JW, Matherne GP, Smith FC, Hoke TR, Koppel R, Gidding SS, Beekman RH 3rd, Grosse SD (2009) Role of pulse oximetry inexamining newborns for congenital heart disease: a scientific statement from the AHA and AAP. Pediatrics 124:823-836

18. Malkin JD, Garber S, Broder MS, Keeler E (2000) Infant mortality and early postpartum discharge. Obstetr Gynecol 96: $183-188$

19. Meberg A, Brügmann-Pieper S, Due R Jr, Eskedal L, Fagerli I, Farstad T, Frøisland DH, Sannes CH, Johansen OJ, Keljalic J, Markestad T, Nygaard EA, Røsvik A, Silberg IE (2008) First day of life pulse oximetry screening to detect congenital heart defects. J Pediatr 152:761-765

20. Mellander M, Sunnegårdh J (2006) Failure to diagnose critical heart malformations in newborns before discharge - an increasing problem? Acta Paediatr 95:407-413

21. O'Donnell CPF, Kamlin COF, Davis PG, Carlin JB, Morley CJ (2007) Clinical assessment of infant colour at delivery. Arch Dis Child Fetal Neonatal Ed 92:F465-F467

22. Reich JD, Connolly B, Bradley G, Littman S, Koeppel W, Lewycky P, Liske M (2008) Reliability of a single pulse oximetry reading as a screening test for congenital heart disease in otherwise asymptomatic newborn infants: the importance of human factors. Pediatr Cardiol 29:371-376

23. Reich JD, Miller S, Brogdon B, Casatelli J, Gompf TC, Huhta JC, Sullivan K (2003) The use of pulse oximetry to detect congenital heart disease. J Pediatr 142:268-272

24. Richmond S, Reay G, Abu Harb M (2002) Routine pulse oximetry in the asymptomatic newborn. Arch Dis Child Fetal Neonatal Ed 87:F83-F88

25. Riede FT, Dähnert I, Schneider P, Möckel A (2009) Pulse oximetry screening at 4 hours of age to detect critical congenital heart defects. Pediatrics 123:e542, author reply e542-e543

26. Rosati E, Chitano G, Dipaola L, De Felice C, Latini G (2005) Indications and limitations for a neonatal pulse oximetry screening of critical congenital heart disease. J Perinat Med 33:455-457

27. Schultz AH, Localio AR, Clark BJ, Ravishankar C, Videon N, Kimmel SE (2008) Epidemiologic features of the presentation of critical congenital heart disease: implications for screening. Pediatrics 121:751-757

28. Sendelbach DM, Jackson GL, Lai SS, Fixler DE, Stehel EK, Engle WD (2008) Pulse oximetry screening at 4 hours of age to detect critical congenital heart defects. Pediatrics 122:e815-e820

29. Thangaratinam S, Daniels J, Ewer AK, Zamora J, Khan KS (2007) Accuracy of pulse oximetry in screening for congenital heart disease in asymptomatic newborns: a systematic review. Arch Dis Child Fetal Neonatal Ed 92:F176-F180

30. Valmari P (2007) Should pulse oximetry be used to screen for congenital heart disease? Arch Dis Child Fetal Neonatal Ed 92: F219-F224

31. Wren C, Reinhardt Z, Khawaja K (2008) Twenty-year trends in diagnosis of life-threatening neonatal cardiovascular malformations. Arch Dis Child Fetal Neonatal Ed 93:F33-F35

32. Wren C, Richmond S, Donaldson L (1999) Presentation of congenital heart disease in infancy: implications for routine examination. Arch Dis Child Fetal Neonatal Ed 80:F49-F53 